M. V. van Hecke - J. M. Dekker · G. Nijpels •

A. C. Moll • R. J. Heine · L. M. Bouter • B. C. P. Polak •

C. D. A. Stehouwer

\title{
Inflammation and endothelial dysfunction are associated with retinopathy: the Hoorn Study
}

Received: 23 December 2004 / Accepted: 24 February 2005 / Published online: 26 May 2005

(C) Springer-Verlag 2005

\begin{abstract}
Aims/hypothesis: The exact pathogenesis of retinopathy in diabetic and non-diabetic individuals is incompletely understood, but may involve chronic low-grade inflammation and dysfunction of the vascular endothelium. The aim of this study was to investigate the association of inflammation and endothelial dysfunction with prevalent retinopathy in individuals with and without type 2 diabetes. Methods: As part of a population-based cohort study, 625 individuals aged 50-74 years, stratified according to age, sex and glucose tolerance status, underwent an extensive physical examination. Retinopathy was assessed by an ophthalmological examination, including funduscopy and two-field $45^{\circ}$ fundus photography with mydriasis in both
\end{abstract}

M. V. van Hecke $(\bowtie) \cdot$ A. C. Moll · B. C. P. Polak

Department of Ophthalmology, VU University Medical Centre,

P.O. Box 7057, 1007 MB Amsterdam, The Netherlands

e-mail: m.vanhecke@vumc.nl

Tel.: +31-20-4444795

Fax: $+31-20-4444745$

M. V. van Hecke · J. M. Dekker · G. Nijpels · A. C. Moll · R. J. Heine - L. M. Bouter - B. C. P. Polak - C. D. A. Stehouwer Institute for Research in Extramural Medicine,

VU University Medical Centre,

Amsterdam, The Netherlands

G. Nijpels

Department of General Practice,

VU University Medical Centre,

Amsterdam, The Netherlands

R. J. Heine

Department of Endocrinology,

VU University Medical Centre,

Amsterdam, The Netherlands

C. D. A. Stehouwer

Department of Internal Medicine,

VU University Medical Centre,

Amsterdam, The Netherlands

C. D. A. Stehouwer

Department of Internal Medicine,

University Hospital Maastricht,

Maastricht, The Netherlands eyes. Levels of C-reactive protein (CRP), soluble intercellular adhesion molecule-1 (sICAM-1), von Willebrand factor, and soluble vascular adhesion molecule-1 (sVCAM-1) were assessed, together with the urinary albumin : creatinine ratio, and the results were combined to obtain summarising $z$ scores for inflammation and endothelial dysfunction. Results: The prevalence of retinopathy was positively associated with tertiles of CRP and sICAM- 1 . When compared with the lowest tertile, the highest tertile of the inflammatory $z$ score was associated with retinopathy in all subjects (odds ratio $[\mathrm{OR}]=2.2,95 \%$ CI $1.2-4.1$, adjusted for age, sex and glucose tolerance status). The highest tertile of the endothelial dysfunction $z$ score was associated with retinopathy among diabetic individuals $(\mathrm{OR}=4.4,95 \% \mathrm{CI}$ 1.2-15.9, adjusted for age and sex) but not in non-diabetic individuals. Additional adjustment for other risk factors, such as systolic and diastolic blood pressure, BMI, total cholesterol and triglycerides, or mutual adjustment of the inflammatory and endothelial dysfunction $z$ scores did not change the results. Conclusions/interpretation: In this study, inflammatory activity and endothelial dysfunction were associated with retinopathy, which suggests their involvement in the pathogenesis of retinopathy.

Keywords Endothelial dysfunction - Inflammation · Retinopathy · Type 2 diabetes

Abbreviations ACR: albumin:creatinine ratio - CRP: C-reactive protein - IGM: impaired glucose metabolism . NGM: normal glucose metabolism - OR: odds ratio sICAM-1: soluble intercellular adhesion molecule-1 . sVCAM-1: soluble vascular adhesion molecule-1 . vWf: von Willebrand factor

\section{Introduction}

Hyperglycaemia, diabetes duration, hypertension, dyslipidaemia and obesity are important risk factors for the development and progression of diabetic retinopathy [1-3]. However, the exact pathogenesis of diabetic retinopathy 
remains unclear. Inflammation and endothelial dysfunction are possible mechanisms, and these may play an important role in the aetiology of diabetic retinopathy [4].

Markers of inflammation and endothelial dysfunction, such as C-reactive protein (CRP), soluble intercellular adhesion molecule-1 (sICAM-1), soluble vascular cell adhesion molecule-1 (sVCAM-1) and von Willebrand factor (vWf), have been associated with macroangiopathy in non-diabetic individuals and in those with type 2 diabetes [5-9]. With respect to microangiopathy, inflammation and endothelial dysfunction have been associated with the development of microalbuminuria $[10,11]$, which itself is strongly associated with retinopathy in type 1 and type 2 diabetes [1214]. Furthermore, levels of sICAM-1 and sVCAM-1 have been reported to be elevated in subjects with diabetic retinopathy [15-17]. Taken together, these observations raise the question of whether inflammation and endothelial dysfunction are involved in the pathogenesis of retinopathy.

To our knowledge, the associations of inflammation and endothelial dysfunction with retinopathy have never been investigated in a population-based study. The aim of the present study was to investigate the associations of inflammation (estimated by levels of CRP and sICAM-1) and endothelial dysfunction (estimated by levels of vWf and sVCAM-1 and the urinary albumin:creatinine ratio $[\mathrm{ACR}]$ ), with retinopathy.

\section{Subjects and methods}

Study design The Hoorn Study is a cohort study of type 2 diabetes and cardiovascular complications in a 50- to 74-year-old general Caucasian population, which started in $1989(n=2484$, response rate $71 \%)$ and has already been extensively described [18]. All subjects, apart from those previously diagnosed with diabetes who were being treated with oral glucose-lowering agents or insulin, underwent an OGTT. All diabetic patients and all persons with a 2-h post-load glucose concentration $\geq 7.5 \mathrm{mmol} / \mathrm{l}$, as well as an age- and sex-stratified random sample of persons with a 2-h post-load glucose concentration of $<7.5 \mathrm{mmol} / \mathrm{l}$, were invited within 4 weeks of their first visit for a second visit to investigate complications related to glucose intolerance (of 709 invited, 631 [89\%] participated) [18]. These subjects underwent a second OGTT (apart from those who already used blood-glucose-lowering agents $[n=67])$. Using the mean value of two OGTTs, patients were divided into three categories on the basis of glucose tolerance: normal glucose metabolism (NGM; $n=258$ ), impaired glucose metabolism (IGM; $n=179$ ) or type 2 diabetes $(n=192)$. The diagnoses were made according to the criteria adopted by the World Health Organization in 1999 (NGM: fasting plasma glucose $<7.0 \mathrm{mmol} / 1$ and 2-h postload plasma glucose $<7.8 \mathrm{mmol} / \mathrm{l}$; IGM: fasting plasma glucose $<7.0 \mathrm{mmol} / \mathrm{l}$ and 2-h post-load plasma glucose 7.8 11.1 ; type 2 diabetes: fasting plasma glucose $\geq 7.0 \mathrm{mmol} / 1$ or 2 -h post-load plasma glucose $\geq 11.1 \mathrm{mmol} / \mathrm{l}$ ) [19]. Subjects treated with oral glucose-lowering agents, insulin or diet were classified as having known diabetes. The sam- pling method used was chosen for reasons of efficiency, because we wished to study a small, but still random, sample in more detail. As the exact sampling procedure is known, we can back-calculate the prevalences in the initial cohort $(n=2484)$ from those in the smaller sample $(n=631)$, as previously described in detail $[18,20]$. Written informed consent was obtained from all participants. The Ethical Review Committee of the VU University Medical Centre (Amsterdam, the Netherlands) approved the Hoorn Study.

Ophthalmological examination At baseline, the retina was examined with funduscopy and/or fundus photography after mydriasis with eye drops containing $0.5 \%$ tropicamide and 5\% phenylephrine. This ophthalmological examination has been extensively described previously [1]. Briefly, funduscopy reports and/or fundus photographs were obtained from 626 participants. The fundus photographs of 148 persons were lost (not associated with age, sex, glucose tolerance category, blood pressure or worst eye visual acuity; data not shown). For one person, both photographs were not gradable and the funduscopy report was incomplete. Thus, 625 individuals were included for further analyses. All photographs $(11 \times 11 \mathrm{~cm})$ were re-graded for retinopathy according to the EURODIAB standards [1]. The EURODIAB classification scheme was used because this uses twofield $45^{\circ}$ fundus photography and standard photographs to grade retinal lesions [21]. Retinopathy was defined as the presence of one or more microaneurysms, haemorrhages, hard exudates, areas of neovascularisation, fibrous proliferation and/or laser coagulation scars in at least one eye. Minimal non-proliferative retinopathy (EURODIAB grade 1) was diagnosed using fundus photography [21]. In each subject the 'worst' eye was graded for retinopathy using fundus photography or funduscopy.

Markers of endothelial dysfunction and inflammation Concentrations of CRP, sICAM-1, vWf and sVCAM-1 were assessed in frozen $\left(-70^{\circ} \mathrm{C}\right)$, heparinised samples of plasma. The mean duration of storage was 8.3 years (range 7.3- 9.4 years). Because of a lack of spare plasma, values for CRP, vWf and sVCAM-1 are missing for 21 subjects and values for sICAM-1 are missing for 23 subjects. The assessment of sVCAM-1, vWf, CRP and sICAM-1 has previously been described $[5,6,22]$. Microalbuminuria was defined as an ACR of $2.0-30.0 \mathrm{mg} / \mathrm{mmol}$ in an earlymorning, first-voided spot urine sample [23]. In a random sample of 174 subjects, the ACR was based on the mean of two measurements [23]. In this study the ACR was used as a continuous measure of urinary albumin excretion.

Additional measurements We measured $\mathrm{HbA}_{1} \mathrm{c}$, blood pressure, total cholesterol, HDL cholesterol, triglycerides, homocysteine, weight, height and body circumference, as described previously $[18,24]$. Subjects with a diastolic blood pressure $\geq 90 \mathrm{mmHg}$, a systolic blood pressure $\geq 140$ $\mathrm{mmHg}$ and/or using antihypertensive medication were considered to have hypertension [25]. The Rose questionnaire was used to determine the subjects' history of cardiovascular disease [18]. 
Statistical analyses Results are presented as means \pm SD, percentages or, in the case of a skewed distribution, as medians with interquartile ranges. Correlations between the independent variables CRP, sICAM-1, vWf and sVCAM-1 were studied and calculated using the partial correlation coefficient.

To create a more robust estimate for inflammation and endothelial dysfunction, we constructed a summarising score for each entity by adding the individual markers together. To this end, an individual $z$ score for every marker of inflammation and endothelial dysfunction was assessed for each subject as follows: (individual value-the mean value for the study population)/standard deviation. Because the regression coefficients of the individual $z$ scores were not significantly different from each other ( $p$ values $>0.05)$, summarising scores could be created. The summarising score for inflammation was calculated as $(z$ score for $\mathrm{CRP}+z$ score for sICAM-1)/2. Similarly, we constructed a summarising score for endothelial dysfunction using $z$ scores for $\mathrm{vWf}$, sVCAM-1 and ACR (used as a continuous marker of urinary albumin excretion). Compared with the individual markers, both summarising scores improved the fit of the model $(p<0.05)$. Because sVCAM-1 and sICAM- 1 may be markers of both inflammation and endothelial dysfunction, we constructed two other scores for inflammation and en- dothelial dysfunction, including sICAM-1 and sVCAM-1 in both scores.

Logistic regression analyses were used to assess the risk of retinopathy associated with baseline variables and markers of inflammation and endothelial dysfunction. Because there was a non-linear relationship between retinopathy and CRP, sICAM-1, vWf, sVCAM-1, urinary ACR, and the summarising scores for inflammatory activity and endothelial dysfunction, tertiles were constructed for these variables, with the lowest tertile used as the reference category. Because of the sampling procedure used, we first adjusted for age, sex and glucose tolerance status (stratification variables), using two dummy variables for age, one for sex and three for glucose tolerance status (for IGM, newly diagnosed diabetes and known diabetes) [18] (crude model). We also adjusted for potential confounders (adjusted model), i.e. variables significantly associated with retinopathy (Table 1). We did not include $\mathrm{HbA}_{1} \mathrm{c}$ in the multivariate model because it is likely that hyperglycaemia causes endothelial dysfunction and inflammation, which means that analyses adjusted for $\mathrm{HbA}_{1} \mathrm{c}$ are probably overadjusted to a certain extent. Furthermore, we did not consider microalbuminuria as a potential confounder, as it was already included in the $z$ score for endothelial dysfunction.

Table 1 Baseline characteristics of the participants according to retinopathy status

\begin{tabular}{|c|c|c|c|c|}
\hline & No retinopathy $(n=540)$ & Retinopathy $(n=85)$ & Difference in risk factor or indicator & OR $(95 \% \mathrm{CI})$ \\
\hline Sex (\% male $)$ & 49 & 40 & Male vs female & $0.74(0.46-1.19)$ \\
\hline Age (years) & $64 \pm 7$ & $65 \pm 7$ & Per 5-year increase & $1.03(0.87-1.22)$ \\
\hline Type 2 diabetes $(\%)$ & 28 & 48 & Versus normal glucose metabolism & $2.33(1.46-3.73)$ \\
\hline $\mathrm{HbA}_{1} \mathrm{c}(\%)$ & $5.80 \pm 1.20$ & $6.66 \pm 1.76$ & Per $1 \%$ increase & $1.28(1.07-1.53)$ \\
\hline Diastolic blood pressure $(\mathrm{mmHg})$ & $82 \pm 10$ & $85 \pm 11$ & Per $10-\mathrm{mmHg}$ increase & $1.32(1.05-1.67)$ \\
\hline Use of antihypertensive drugs (\%) & 27 & 34 & Yes vs no & $1.00(0.59-1.68)$ \\
\hline WHR & $0.92 \pm 0.09$ & $0.93 \pm 0.08$ & Per 0.01 increase & $1.01(0.98-1.05)$ \\
\hline Waist circumference $(\mathrm{cm})$ & $93.5 \pm 11.2$ & $97.1 \pm 11.0$ & Per 5-cm increase & $1.12(0.99-1.26)$ \\
\hline BMI $\left(\mathrm{kg} / \mathrm{m}^{2}\right)$ & $27.0 \pm 3.8$ & $28.7 \pm 4.7$ & Per $5-\mathrm{kg} / \mathrm{m}^{2}$ increase & $1.36(1.02-1.83)$ \\
\hline History of cardiovascular disease $(\%)$ & 22 & 28 & Yes vs no & $1.16(0.68-1.97)$ \\
\hline $\mathrm{ACR}(\mathrm{mg} / \mathrm{mmol})$ & $0.84(0.58-1.30)$ & $1.07(0.60-2.58)$ & Per $10 \%$ increase & $2.01(1.45-2.79)$ \\
\hline Microalbuminuria (\%) & 10 & 25 & Yes vs no & $2.07(1.11-3.86)$ \\
\hline vWf (IU/ml) & $1.37 \pm 0.71$ & $1.40 \pm 0.66$ & Per $10 \%$ increase & $0.99(0.95-1.04)$ \\
\hline CRP (mg/l) & $1.69(0.80-3.61)$ & $2.39(1.16-4.72)$ & Per $10 \%$ increase & $1.01(0.99-1.03)$ \\
\hline sVCAM-1 (ng/ml) & $1298(1080-1583)$ & $1355(1157-1669)$ & Per $10 \%$ increase & $1.02(0.95-1.10)$ \\
\hline sICAM-1 (ng/ml) & $462(370-541)$ & $500(413-601)$ & Per $10 \%$ increase & $1.07(0.99-1.15)$ \\
\hline Inflammatory marker $z$ score & $-0.03 \pm 0.77$ & $0.18 \pm 0.73$ & Per 1-unit increase & $1.16(0.88-1.54)$ \\
\hline Endothelial function marker $z$ score & $-0.03 \pm 0.57$ & $0.21 \pm 1.14$ & Per 1-unit increase & $1.24(0.90-1.71)$ \\
\hline
\end{tabular}

Data are presented as percentages, means $\pm \mathrm{SD}$ or, in the case of a skewed distribution, as medians (interquartile ranges). Odds ratios (OR) with $95 \%$ CIs were obtained by logistic regression after adjustment for age, sex and glucose tolerance status (unless it was the variable under consideration) 


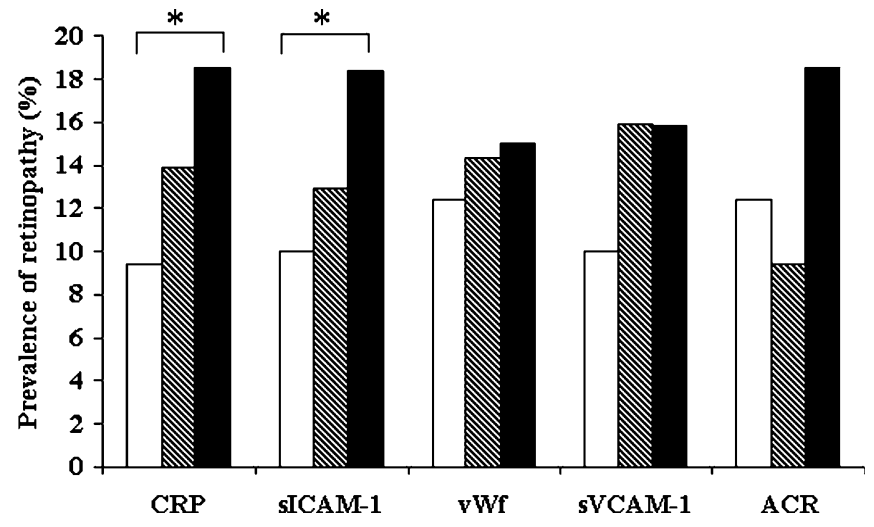

Fig. 1 Prevalence of retinopathy according to tertiles (low, white bars; medium, hatched bars; high, black bars) of CRP, sICAM-1, vWf, sVCAM-1 and ACR. ${ }^{*} p<0.05$ for trend

We tested for interactions of diabetes status (newly discovered and known) with markers of endothelial dysfunction and inflammation (diabetes yes/no $\times$ marker tertile). Data are presented as odds ratios (ORs) with $95 \%$ CIs. All analyses were performed using SPSS statistical software, Version 10.1 for Windows 95 (SPSS, Chicago, IL, USA). A $p$ value of less than 0.05 was considered statistically significant.

\section{Results}

Retinopathy was present in $85(13.6 \%)$ of the 625 individuals assessed by funduscopy and/or fundus photography. Table 1 shows the baseline characteristics of the subjects according to retinopathy status, and the odds ratios for retinopathy for each risk factor or risk indicator, adjusted for age, sex and glucose tolerance status (unless it was the variable under consideration). Type 2 diabetes, $\mathrm{HbA}_{1} \mathrm{c}$, systolic and diastolic blood pressure, BMI, total cholesterol, triglycerides and microalbuminuria were significantly associated with retinopathy. Among the non-diabetic subjects, systolic and diastolic blood pressures were significantly higher in subjects with retinopathy than in those without retinopathy ( $p=0.05$ and $p=0.03$, respectively). However, among the diabetic subjects, only systolic blood pressure was significantly higher in subjects with retinopathy ( $p=$ 0.01) (data not shown).
Levels of CRP, sICAM-1, vWf and sVCAM-1 were weakly correlated with each other. The age-, sex- and glucose-tolerance-adjusted correlation coefficient between sICAM-1 and sVCAM-1 was $0.31(p<0.001)$; all other factors were less strongly correlated (data not shown).

The prevalence of retinopathy increased across increasing tertiles of CRP and sICAM-1 (Fig. 1). The highest tertiles of CRP and sICAM-1 were both associated with retinopathy, with non-significant odds ratios of 1.7 ( $p=0.11$ and 0.08 respectively) compared with the lowest tertiles, whereas the highest tertile of the inflammatory marker $z$ score was significantly associated with retinopathy $(\mathrm{OR}=2.2,95 \%$ CI $1.2-4.1, p=0.01)$, after adjustment for age, sex and glucose tolerance status (Table 2). These associations were present in both non-diabetic and diabetic individuals. Additional adjustment for systolic and diastolic blood pressure, BMI, total cholesterol and triglycerides did not materially change these results.

There was a significant interaction $(p=0.005)$ between endothelial dysfunction and diabetes status; thus, the results are shown separately for individuals with and those without diabetes. Table 3 shows that, after adjustment for sex and age, the highest tertile of the endothelial dysfunction marker $z$ score was significantly associated with retinopathy among diabetic subjects $(\mathrm{OR}=4.4,95 \% \mathrm{CI} 1.2$ $15.9, p=0.02)$ but not among non-diabetic subjects $(\mathrm{OR}=$ $0.5,95 \%$ CI $0.2-1.3, p=0.17$ ). The highest tertile of sVCAM-1 was also significantly associated with retinopathy in diabetic subjects $(\mathrm{OR}=4.5,95 \%$ CI $1.4-14.3$, $p=0.01$ ), whereas the highest tertiles of $\mathrm{vWf}$ and ACR were associated with a non-significant elevated risk of retinopathy among diaLbetic subjects $(p=0.08$ and 0.46 , respectively). Additional adjustment for systolic and diastolic blood pressure, BMI, total cholesterol and triglycerides did not considerably change these associations.

Additional analyses It has been argued that SVCAM-1 may also be considered an indicator of inflammation and sICAM-1 an indicator of endothelial dysfunction. However, neither the addition of sVCAM-1 to the inflammatory marker $z$ score nor the addition of sICAM-1 to the endothelial dysfunction marker $z$ score altered the results (data not shown).

In addition, the values for the association between the endothelial dysfunction marker $z$ score and retinopathy
Table 2 Odds ratios $(95 \%$ CIs) for retinopathy associated with markers of inflammation in all subjects

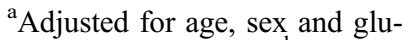
cose tolerance status; ${ }^{b}$ as crude model with additional adjustment for systolic and diastolic blood pressure, BMI, total cholesterol and triglycerides; ${ }^{\mathrm{c}} z$ score for CRP+sICAM-1. $n / N$, number of cases/total number
}

\begin{tabular}{lclll}
\hline & Tertiles & $n / N^{\mathrm{c}}$ & $\begin{array}{l}\text { Crude model }^{\mathrm{a}} \\
\text { OR }(95 \% \mathrm{CI})\end{array}$ & $\begin{array}{l}\text { Adjusted model }^{\mathrm{b}} \\
\text { OR }(95 \% \mathrm{CI})\end{array}$ \\
\hline CRP (mg/l) & $0.02-1.10$ & $19 / 203$ & 1 & 1 \\
& $1.11-2.84$ & $28 / 201$ & $1.32(0.70-2.49)$ & $1.21(0.64-2.31)$ \\
& $2.85-35.18$ & $37 / 200$ & $1.66(0.89-3.08)$ & $1.41(0.75-2.68)$ \\
sICAM-1 (ng/ml) & $97.2-410.2$ & $20 / 200$ & 1 & 1 \\
& $410.4-512.7$ & $26 / 201$ & $1.17(0.62-2.20)$ & $1.19(0.63-2.24)$ \\
& $512.8-1793.6$ & $37 / 201$ & $1.71(0.94-3.11)$ & $1.50(0.81-2.75)$ \\
Inflammatory marker $z$ score $^{\mathrm{c}}$ & -1.35 to -0.39 & $17 / 200$ & 1 & 1 \\
& -0.38 to 0.09 & $25 / 201$ & $1.40(0.72-2.70)$ & $1.32(0.68-2.58)$ \\
& 0.10 to 4.90 & $41 / 201$ & $2.22(1.19-4.12)$ & $1.96(1.03-3.72)$ \\
\hline
\end{tabular}


were adjusted for the inflammatory marker $z$ score and vice versa. Neither adjustment altered the results presented above (data not shown).

Values were also adjusted for prior cardiovascular disease, $\mathrm{HbA}_{1} \mathrm{c}$, and antihypertensive treatment (data not shown). Adjustment for $\mathrm{HbA}_{1} \mathrm{c}$ reduced the odds ratio for the highest tertile of the inflammatory marker $z$ score to 1.8 (95\% CI $0.9-3.3)$, and the odds ratios for the endothelial function marker $z$ score were 0.4 (95\% CI 0.2-1.2) and $4.45(95 \%$ CI 1.13-17.60) in non-diabetic and diabetic subjects, respectively. Furthermore, additional adjustment for prior CVD and anti-hypertensive treatment did not change the results $(p>0.05$ for the summarised scores for inflammation and endothelial dysfunction).

\section{Discussion}

In the present study, inflammatory activity was associated with retinopathy in both diabetic and non-diabetic individuals, and endothelial dysfunction was associated with retinopathy in diabetic individuals, but not in non-diabetic individuals. These associations were independent of other risk factors for retinopathy and of each other, and suggest the involvement of inflammatory processes and endothelial dysfunction in the pathogenesis of this complication.

This is the first population-based study to show that inflammatory activity, estimated by a $z$ score for CRP and sICAM-1, is associated with retinopathy in diabetic individuals, as well as in non-diabetic individuals. In previous studies, inflammation has been shown to play an important role in atherosclerosis [26]. Inflammatory activity is higher in individuals with type 1 or type 2 diabetes than in their non-diabetic peers $[9,27]$, and inflammatory activity in diabetes is associated with macrovascular disease $[5,6,9,11$, $22,28,29]$ and microangiopathy $[11,15,30]$. Our data are consistent with these results, and show that inflammatory activity may also be important in the pathogenesis of retinopathy in non-diabetic individuals.

Endothelial dysfunction was associated with a 5-fold higher risk of retinopathy in diabetic subjects; there was no clear association in non-diabetic subjects. It has been hypothesised that endothelial dysfunction is a key feature of the pathogenesis of atherosclerosis in diabetic and nondiabetic individuals $[26,31]$ and also of the pathogenesis of diabetic microangiopathy, especially nephropathy $[4,10$, $11,32]$. However, relatively few studies have reported on the association between markers of endothelial function and retinopathy. Individuals with diabetic retinopathy (type 1 and type 2) have been shown to have higher levels of sVCAM-1 than those without retinopathy [15-17]. Results on the relationship between $\mathrm{vWf}$ and retinopathy have been conflicting. On the one hand, in early-stage retinopathy in type 1 diabetes, vWf has been associated with a prolonged retinal circulation time and reduced retinal blood flow, which may promote stasis in the retinal circulation, leading to hypoxaemia [33]. On the other hand, vWf was not found to be related to the presence and development of very early retinopathy in type 1 diabetes [34]. Microalbuminuria is 
often regarded as a marker of generalised endothelial dysfunction [4], and is strongly associated with retinopathy in both type 1 and type 2 diabetes [12-14]. Microalbuminuria was also strongly associated with retinopathy in our study. However, within the normal range, tertiles of ACR were not strongly associated with retinopathy. Taken together, the results of previous work and the present study are consistent with a role for endothelial dysfunction in the pathogenesis of diabetic retinopathy. Although associations with individual markers (vWf, sVCAM-1, ACR) have not always been consistent, this may partly be due to the considerable biological variation inherent in the (usually single) measurement of such markers. A major advantage of the use of an endothelial function marker $z$ score is that it limits the influence of biological variation, giving a more precise estimate. Indeed, our data suggest that the association between endothelial dysfunction and retinopathy in type 2 diabetes is relatively strong.

Changes in the retina, similar to those that occur in diabetic retinopathy, can also be found in non-diabetic individuals $[1,35,36]$, although such changes do not reach the stage of diabetic macular oedema or proliferative retinopathy. Risk factors for these early phases of retinopathy in non-diabetic subjects were hypertension, IGT status, dyslipidaemia and obesity $[1,35]$. The present results indicate that inflammatory activity may also contribute to these early phases.

Our results are supported by recent experimental studies, mainly on rodent retinas, which have shown that retinopathy is associated with low-grade chronic inflammation $[37,38]$. It has been hypothesised that vascular endothelial growth factor (VEGF), a molecule closely related to the pathogenesis of retinopathy, can trigger early retinal inflammation by inducing the expression and upregulation of sICAM-1 [39]. Increased levels of adhesion molecules, which cause leucocyte adhesion and stasis in the retinal vasculature $[40,41]$, and increased permeability of the endothelium, may be the initial step in the complex pathology of retinopathy [42]. Leucostasis could produce temporary ischaemia upstream and subsequent reperfusion, which may lead to endothelial cell injury and death through the formation of reactive oxygen species [43].

The associations of inflammation and endothelial dysfunction with retinopathy were independent of each other, suggesting that inflammation and endothelial dysfunction play separate roles in the pathogenesis of retinopathy. Because of the cross-sectional design of this study, it does not provide information on the inter-relationship between inflammation and endothelial dysfunction, except that adhesion molecules were weakly correlated. Nevertheless, experimental evidence and observational data in humans show that inflammation can induce endothelial dysfunction and vice versa, thus potentially creating a vicious circle [11].

One of the strengths of this study was that we investigated several markers of inflammation and endothelial dysfunction simultaneously. We created $z$ scores, producing statistically robust summarising estimates of both inflammation and endothelial dysfunction. Inflammation and endothelial dysfunction are complex entities that cannot be accurately reflected by a single marker. Since sICAM-1 and SVCAM-1 both contribute to leucocyte adhesion and transmigration through the endothelium following activation in response to inflammatory cytokines [44, 45], sICAM-1 and sVCAM-1 may be markers of both inflammation and endothelial dysfunction. For this reason, we constructed two summarising scores for inflammation, one with and one without SVCAM-1, and two summarizing scores for endothelial dysfunction, one with and one without SICAM-1. This did not materially change the results.

A main limitation of this study was the relatively small sample size, which resulted in wide confidence intervals. Furthermore, this power problem prevented the stratification of the subjects according to different stages of retinopathy. The majority of individuals with retinopathy had minimal non-proliferative retinopathy $(n=73)$, indicating that inflammatory activity and endothelial dysfunction are involved in even minimal disorders of the retinal vasculature. The associations were, if anything, stronger in the small group of participants $(n=12)$ with worse than minimal non-proliferative retinopathy (data not shown).

We conclude that inflammatory activity and endothelial dysfunction are potentially important contributors to retinopathy. These data provide a basis for testing the effects of treatment aimed at decreasing inflammatory activity and improving endothelial function as a means of preventing or limiting the progression of retinopathy.

\section{References}

1. van Leiden HA, Dekker JM, Moll AC et al (2002) Blood pressure, lipids, and obesity are associated with retinopathy: the Hoorn study. Diabetes Care 25:1320-1325

2. Nagi DK, Pettitt DJ, Bennett PH, Klein R, Knowler WC (1997) Diabetic retinopathy assessed by fundus photography in Pima Indians with impaired glucose tolerance and NIDDM. Diabet Med 14:449-456

3. Stratton IM, Kohner EM, Aldington SJ et al (2001) UKPDS 50: risk factors for incidence and progression of retinopathy in Type II diabetes over 6 years from diagnosis. Diabetologia 44:156-163

4. Stehouwer CD, Lambert J, Donker AJ, van Hinsbergh VW (1997) Endothelial dysfunction and pathogenesis of diabetic angiopathy. Cardiovasc Res 34:55-68

5. Jager A, van Hinsbergh VW, Kostense PJ et al (2000) Increased levels of soluble vascular cell adhesion molecule 1 are associated with risk of cardiovascular mortality in type 2 diabetes: the Hoorn study. Diabetes 49:485-491

6. Jager A, van Hinsbergh VW, Kostense PJ et al (1999) Von Willebrand factor, C-reactive protein, and 5-year mortality in diabetic and nondiabetic subjects: the Hoorn study. Arterioscler Thromb Vasc Biol 19:3071-3078

7. Otsuki M, Hashimoto K, Morimoto Y, Kishimoto T, Kasayama S (1997) Circulating vascular cell adhesion molecule-1 (VCAM-1) in atherosclerotic NIDDM patients. Diabetes 46:2096-2101

8. Hwang SJ, Ballantyne CM, Sharrett AR et al (1997) Circulating adhesion molecules VCAM-1, ICAM-1, and E-selectin in carotid atherosclerosis and incident coronary heart disease cases: the Atherosclerosis Risk In Communities (ARIC) study. Circulation 96:4219-4225

9. Kado S, Nagata N (1999) Circulating intercellular adhesion molecule-1, vascular cell adhesion molecule-1, and E-selectin in patients with type 2 diabetes mellitus. Diabetes Res Clin Pract 46:143-148 
10. Jager A, van Hinsbergh VW, Kostense PJ et al (2002) C-reactive protein and soluble vascular cell adhesion molecule-1 are associated with elevated urinary albumin excretion but do not explain its link with cardiovascular risk. Arterioscler Thromb Vasc Biol 22:593-598

11. Stehouwer CD, Gall MA, Twisk JW, Knudsen E, Emeis JJ, Parving HH (2002) Increased urinary albumin excretion, endothelial dysfunction, and chronic low-grade inflammation in type 2 diabetes: progressive, interrelated, and independently associated with risk of death. Diabetes 51:1157-1165

12. Cruickshanks KJ, Ritter LL, Klein R, Moss SE (1993) The association of microalbuminuria with diabetic retinopathy. The Wisconsin Epidemiologic Study of Diabetic Retinopathy. Ophthalmology 100:862-867

13. Wirta O, Pasternack A, Mustonen J, Laippala P, Lahde Y (1999) Retinopathy is independently related to microalbuminuria in type 2 diabetes mellitus. Clin Nephrol 51:329-334

14. Crimi S, Cipolli D, Infantone E, Infantone L, Lunetta M (1995) Microalbuminuria and severity of diabetic retinopathy in type 1 diabetic patients: association and relationship with some risk factors. Diabetes Metab 21:440-445

15. Matsumoto K, Sera Y, Ueki Y, Inukai G, Niiro E, Miyake S (2002) Comparison of serum concentrations of soluble adhesion molecules in diabetic microangiopathy and macroangiopathy. Diabet Med 19:822-826

16. Fasching P, Veitl M, Rohac M et al (1996) Elevated concentrations of circulating adhesion molecules and their association with microvascular complications in insulin-dependent diabetes mellitus. J Clin Endocrinol Metab 81:4313-4317

17. Koga M, Otsuki M, Kubo M, Hashimoto J, Kasayama S (1998) Relationship between circulating vascular cell adhesion molecule-1 and microvascular complications in type 2 diabetes mellitus. Diabet Med 15:661-667

18. Beks PJ, Mackaay AJ, de Neeling JN, de Vries H, Bouter LM, Heine RJ (1995) Peripheral arterial disease in relation to glycaemic level in an elderly Caucasian population: the Hoorn study. Diabetologia 38:86-96

19. World Health Organization (1999). Definition, diagnosis and classification of diabetes mellitus and its complications. Report of a WHO consultation, Part 1: diagnosis and classification of diabetes mellitus. World Health Organization, Geneva

20. Hoogeveen EK, Kostense PJ, Beks PJ et al (1998) Hyperhomocysteinemia is associated with an increased risk of cardiovascular disease, especially in non-insulin-dependent diabetes mellitus: a population-based study. Arterioscler Thromb Vasc Biol 18:133-138

21. Aldington SJ, Kohner EM, Meuer S, Klein R, Sjolie AK (1995) Methodology for retinal photography and assessment of diabetic retinopathy: the EURODIAB IDDM complications study. Diabetologia 38:437-444

22. Becker A, van Hinsbergh VW, Jager A et al (2002) Why is soluble intercellular adhesion molecule-1 related to cardiovascular mortality? Eur J Clin Invest 32:1-8

23. Jager A, Kostense PJ, Ruhe HG et al (1999) Microalbuminuria and peripheral arterial disease are independent predictors of cardiovascular and all-cause mortality, especially among hypertensive subjects: five-year follow-up of the Hoorn study. Arterioscler Thromb Vasc Biol 19:617-624

24. Hoogeveen EK, Kostense PJ, Eysink PE et al (2000) Hyperhomocysteinemia is associated with the presence of retinopathy in type 2 diabetes mellitus: the Hoorn study. Arch Intern Med 160:2984-2990

25. Anonymous (1997) The sixth report of the Joint National Committee on prevention, detection, evaluation, and treatment of high blood pressure. Arch Intern Med 157:2413-2446

26. Ross R (1999) Atherosclerosis - an inflammatory disease. N Engl J Med 340:115-126
27. Schalkwijk CG, Poland DC, van Dijk W et al (1999) Plasma concentration of C-reactive protein is increased in type I diabetic patients without clinical macroangiopathy and correlates with markers of endothelial dysfunction: evidence for chronic inflammation. Diabetologia 42:351-357

28. Jude EB, Douglas JT, Anderson SG, Young MJ, Boulton AJ (2002) Circulating cellular adhesion molecules ICAM-1, VCAM-1, P- and E-selectin in the prediction of cardiovascular disease in diabetes mellitus. Eur J Intern Med 13:185-189

29. van der Meer IM, de Maat MP, Bots ML et al (2002) Inflammatory mediators and cell adhesion molecules as indicators of severity of atherosclerosis: the Rotterdam Study. Arterioscler Thromb Vasc Biol 22:838-842

30. McLeod DS, Lefer DJ, Merges C, Lutty GA (1995) Enhanced expression of intracellular adhesion molecule-1 and P-selectin in the diabetic human retina and choroid. Am J Pathol 147:642653

31. Bonetti PO, Lerman LO, Lerman A (2003) Endothelial dysfunction: a marker of atherosclerotic risk. Arterioscler Thromb Vasc Biol 23:168-175

32. Stehouwer CD, Fischer HR, van Kuijk AW, Polak BC, Donker AJ (1995) Endothelial dysfunction precedes development of microalbuminuria in IDDM. Diabetes 44:561-564

33. Feng D, Bursell SE, Clermont AC et al (2000) von Willebrand factor and retinal circulation in early-stage retinopathy of type 1 diabetes. Diabetes Care 23:1694-1698

34. Stehouwer CD, Zellenrath P, Polak BC et al (1992) von Willebrand factor and early diabetic retinopathy: no evidence for a relationship in patients with type 1 (insulin-dependent) diabetes mellitus and normal urinary albumin excretion. Diabetologia 35:555-559

35. Rajala U, Laakso M, Qiao Q, Keinanen-Kiukaanniemi S (1998) Prevalence of retinopathy in people with diabetes, impaired glucose tolerance, and normal glucose tolerance. Diabetes Care 21:1664-1669

36. Yu T, Mitchell P, Berry G, Li W, Wang JJ (1998) Retinopathy in older persons without diabetes and its relationship to hypertension. Arch Ophthalmol 116:83-89

37. Adamis AP (2002) Is diabetic retinopathy an inflammatory disease? Br J Ophthalmol 86:363-365

38. Gardner TW, Antonetti DA, Barber AJ, LaNoue KF, Levison SW (2002) Diabetic retinopathy: more than meets the eye. Surv Ophthalmol 47(Suppl 2):S253-S262

39. Joussen AM, Poulaki V, Qin W et al (2002) Retinal vascular endothelial growth factor induces intercellular adhesion molecule-1 and endothelial nitric oxide synthase expression and initiates early diabetic retinal leukocyte adhesion in vivo. Am J Pathol 160:501-509

40. Barouch FC, Miyamoto K, Allport JR et al (2000) Integrinmediated neutrophil adhesion and retinal leukostasis in diabetes. Invest Ophthalmol Vis Sci 41:1153-1158

41. Miyamoto K, Khosrof S, Bursell SE et al (1999) Prevention of leukostasis and vascular leakage in streptozotocin-induced diabetic retinopathy via intercellular adhesion molecule-1 inhibition. Proc Natl Acad Sci U S A 96:10836-10841

42. Olson JA, Whitelaw CM, McHardy KC, Pearson DW, Forrester JV (1997) Soluble leucocyte adhesion molecules in diabetic retinopathy stimulate retinal capillary endothelial cell migration. Diabetologia 40:1166-1171

43. Kohner EM, Patel V, Rassam SM (1995) Role of blood flow and impaired autoregulation in the pathogenesis of diabetic retinopathy. Diabetes 44:603-607

44. Blake GJ, Ridker PM (2001) Novel clinical markers of vascular wall inflammation. Circ Res 89:763-771

45. Meigs JB, Hu FB, Rifai N, Manson JE (2004) Biomarkers of endothelial dysfunction and risk of type 2 diabetes mellitus. JAMA 291:1978-1986 